\title{
Decapping complex is essential for functional P-body formation and is buffered by nuclear localization
}

Kiril Tishinov and Anne Spang

Biozentrum, University of Basel, Klingelbergstrasse 70, $\mathrm{CH}-4056$ Basel, Switzerland

Correspondence:

Anne Spang

University of Basel

Klingelbergstrasse 70

$\mathrm{CH}-4056$ Basel

Switzerland

anne.spang@unibas.ch

Keywords: mRNA, nucleo-cytoplasmic transport, processing bodies, membrane-less granules, phase separation, endoplasmic reticulum 


\section{Abstract (149 words)}

mRNA decay is a key step in regulating the cellular proteome. Cytoplasmic mRNA is largely turned over in processing bodies (P-bodies). P-body units assemble to form Pbody granules under stress conditions. How this assembly is regulated, however, remains still poorly understood. Here, we show that the translational repressor Scd6 and the decapping stimulator Edc3 act partially redundantly in P-body assembly by capturing the Dcp1/2 decapping complex and preventing it from becoming imported into the nucleus by the karyopherin $ß$ Kap95. Nuclear Dcp1/2 does not drive mRNA decay and might be stored there as a ready releasable pool, indicating a dynamic equilibrium between cytoplasmic and nuclear Dcp1/2. Cytoplasmic Dcp1/2 is linked to Dhh1 via Edc3 and Scd6. Functional P-bodies are present at the endoplasmic reticulum where Dcp2 potentially acts to increase the local concentration of Dhh1 through interaction with Scd6 and Edc3 to drive phase separation and hence P-body formation. 


\section{Introduction}

Translational attenuation is among the first lines of defense when a cell encounters stress. Ribosomes will release mRNA and most of the mRNA is captured into processing bodies (P-bodies), which form very quickly, within five minutes after stress encounter. The fast formation of P-bodies might be explained by the notion that the release of mRNAs and their capture into PBs is coordinated in two ways. First, a subset of PB components such as the 5' exonuclease, Xrn1, is associated with polysomes and second, regulators of translation such as Scp160 and Bfr1 negatively regulate PB formation (Weidner et al., 2014; Tesina et al., 2019). Moreover, PBs contain the translational repressor Scd6, which can sequester elF4G (Nissan et al., 2010; Rajyaguru, She and Parker, 2012). P-bodies were initially thought to represent an mRNA decay compartment (Sheth and Parker, 2003). This decay requires removal of the 5' 7-mG cap by the decapping complex Dcp1/2. The decapping activity is stimulated by DEAD box helicase Dhh1/DDX6 and the RNA binding protein Pat1 (Nissan et al., 2010). Recent data, however, provide evidence that P-bodies do not only act as decay compartments but are also mRNA storage organelles and that the fate of an mRNA in PBs is dependent of the type of stressor (Wang et al., 2018; Luo et al., 2020). But not only the fate of mRNAs in PBs is stress-dependent, also the morphology of PBs can vary according to the stress. For example, under glucose starvation, usually 1-2 large P-bodies are observed, while hyperosmotic stress and defects in the secretory pathway induce numerous smaller P-bodies (Kilchert et al., 2010). The different morphologies also suggest that the protein composition of the Pbodies might be dependent on the stressor. Although most of the research on P-bodies has been performed in Saccharomyces cerevisiae, because of the evolutionary conservation of the of the P-body components (Fig. 1A) and its functions, the results obtained in yeast are highly relevant for all metazoans.

P-bodies are membrane-less organelles. Some of the protein components contain unstructured regions or a RecA fold that are able, together with RNA to engage in liquid-liquid phase separation. Indeed, recently it was shown that Dhh1/DDX6 could drive phase separation in vitro and in vivo (Hondele et al., 2019). Other proteins, such Pat1, Edc3 and Scd6 have also been shown to contribute to P-body assembly under at least some stress conditions (Decker, Teixeira and Parker, 2007; Teixeira and Parker, 2007; Kilchert et al., 2010; Sachdev et al., 2019). It appears as if Dhh1 and 
Pat1 act together, while Scd6 and Edc3 seem to have partially redundant functions in P-body formation (Coller and Parker, 2005; Decourty et al., 2008; Nissan et al., 2010). Thus, P-body assembly and function might be regulated through different pathways, consistent with the findings that a variety of kinases can regulate granule assembly (Yoon, Choi and Parker, 2010; Ramachandran, Shah and Herman, 2011). While under stress P-bodies are easily detected by light microscopy, they are essentially undetectable in unstressed cells. Yet, smaller P-body degradative units exist in unstressed cells as the major RNA degradation pathway in yeast is Xrn1-dependent, which acts in P-bodies (Parker, 2012). While the pathway by which Dhh1/DDX6 and Pat1 drive P-body formation is relatively well understood, information about the Scd6/Edc3-dependent pathway is still scarce.

Therefore, we decided to analyze the role of Scd6 and Edc3 in P-body assembly. We found that both proteins are required to retain the decapping complex in the cytoplasm. In an edc3 $\Delta$ scd6 $\Delta$ mutant, Dcp1 and Dcp2 accumulated in the nucleus through active import by Kap95. Nuclear localized Dcp1/Dcp2, however, did not drive nuclear mRNA decay. We propose that the nuclear decapping complex acts as a reservoir to regulate mRNA decay and decapping activity in the cytoplasm. We show furthermore that P-body assembly happens primarily on the endoplasmic reticulum (ER) and that in this process Edc3 and Scd6 link the decapping complex to Dhh1 (Fig.1B). Therefore, Dcp2 is also essential for P-body formation. We envisage that Dcp1/Dcp2 acts to increase the necessary critical concentration of Dhh1 at the ER to drive phase separation.

\section{Results}

\section{Concomitant loss of Scd6 and Edc3 blocks P-body assembly and drives nuclear accumulation of Dcp2}

Previous studies have shown that the individual deletions of EDC3 and SCD6 only partially affect PB formation (Decker, Teixeira and Parker, 2007; Kilchert et al., 2010; Rajyaguru, She and Parker, 2012) and are dispensable for growth (Kshirsagar and Parker, 2004; Decourty et al., 2008). However, since they might have partially overlapping functions, we generated an edc3 $\Delta s c d 6 \Delta$ double mutant and assessed its ability to form P-bodies under stress using Dcp2-GFP as a marker (Fig. 1C). As 
observed previously, deletion of either SCD6 or EDC3 affected P-body formation under hypoosmotic stress and to a somewhat lesser extent under starvation (Fig. 1CE) (Kilchert et al., 2010). This effect was strongly exacerbated in the double mutant, consistent with the notion of a redundant function in P-body granule formation.

Surprisingly, we also observed an accumulation of Dcp2-GFP in the nucleus in the single mutants, which was again strongly increased in the double mutant under normal growth conditions (Fig.1 C and F). In fact, in edc3s scd6s cells, the nuclear Dcp2 localization was maintained even under stress conditions, while this effect was less noticeable in edc3s or scd6s cells. These data suggest that there might be a correlation between Dcp2 localization and the ability of the cell to form P-body granules.

Next, we asked whether the nuclear accumulation was specific for Dcp2 or whether other P-body components would behave in a similar manner in the absence of Edc3 and Scd6. While Dcp1, which is part of the decapping complex, acted similarly to Dcp2, the exonuclease Xrn1 and the Lsm-associated protein Pat1 remained cytoplasmic (Fig. 1G-I). Therefore, loss of Edc3 and Scd6 causes the selective nuclear accumulation of the decapping complex. Nevertheless, Pat1 had been shown previously to become nuclear localized in an Ism1s mutant (Teixeira and Parker, 2007). In addition, in mammalian cells, Pat1's interaction with both the splicing machinery in the cell nucleus and cytoplasmic P-bodies has been demonstrated (Vindry et al., 2017). Yet, Dcp2 was not enriched in the nucleus under the same conditions (Fig. 1I). Our data indicate that at least two independent pathways exist to control the cytoplasmic/nuclear distribution of a subset of P-body components.

\section{Dcp1/Dcp2 requires active import by Kap95 for nuclear localization}

It is conceivable that Dcp2 depends on Dcp1 to be localized to the nucleus in edc3s $s c d 6 \Delta$ cells. To test this possibility, we generated a triple deletion edc3 $\operatorname{scd} 6 \Delta d c p 1 \Delta$. In this strain, Dcp2 still reached the nucleus (Fig. 2A), indicating that nuclear import does not depend on the assembled decapping complex and that Dcp2 itself must contain a nuclear import signal. We analyzed the Dcp2 sequence with the NLStradamus model (Nguyen Ba et al., 2009) for NLS prediction and found three potential monopartite NLSs in the amino acid regions 458-467, 562-576 and 687-708. The last region was also identified using cNLS Mapper, a web-based application for 
prediction of importin substrates (Kosugi et al., 2009), as a part of a moderately strong bipartite NLS spanning over amino acids 673-707. The presence of putative NLSs in the Dcp2 sequence suggests the import might be mediated by the karyopherin $\alpha / \beta$ (Kap60/Kap95) complex. Both KAP60 and KAP95 are essential for cell viability. Therefore, we tagged KAP95 C-terminally with an auxin-inducible degron (AID) in the edc3 $3 \operatorname{scd} 6 \Delta$ background to acutely deplete Kap95 upon addition of auxin. We observed, however, that import of Dcp2-GFP into the nucleus was already impaired in the absence of auxin, indicating that the addition of the degron resulted in a hypomorphic kap95 allele (Fig. 2B and C). Therefore, we conclude that the nuclear localization of Dcp2 is dependent on karyopherin ß.

\section{The decapping complex is stored in the nucleus as a readily releasable pool}

Next, we asked what could be the role of the decapping complex in the nucleus. Dcp1/2 could potentially decap mRNAs already in the nucleus and drive their decay there. Alternatively, the cytoplasmic concentration of active decapping complex might be tightly controlled and the nucleus would only serve as a storage space for any extra decapping complex. First, we explored a potential nuclear function of Dcp2. For this, we overexpressed GFP-tagged Dcp2 or a catalytically dead Dcp2 (Dcp2 ${ }^{\mathrm{CD}}$ ) (Van Dijk et al., 2002) with or without the strong SV40 nuclear localization signal (NLS ${ }^{S 40}$ ) (Fig. $2 \mathrm{D}$ and $\mathrm{E}$ ). While high levels of $\mathrm{Dcp} 2^{\mathrm{CD}}$ reduced cellular fitness over a range of temperatures, confining Dcp2 ${ }^{\mathrm{CD}}$ to the nucleus, rescued this phenotype. Likewise, a high nuclear concentration of Dcp2 was not toxic (Fig. 2F). Our data suggest that Dcp2 has essential decapping functions in the cytoplasm but not in the nucleus. Moreover, high levels of nuclear Dcp2 are well tolerated, suggesting that Dcp2 may not be active in the nucleus.

Decapped RNA is unstable in the nucleus (Kufel et al., 2004). Our results above indicate that Dcp2 should not enhance RNA degradation in the nucleus. To this end, we carried out an mRNA degradation assay in a nup145 $\Delta \mathrm{C}$ mutant, which is deficient for mRNA nuclear export at the restrictive temperature (Kufel et al., 2004). Logarithmically growing cells were first deprived of methionine at the permissive temperature $\left(23^{\circ} \mathrm{C}\right)$ to induce the expression of the MET3 gene and then shifted to the restrictive temperature $\left(37^{\circ} \mathrm{C}\right)$ to block nuclear mRNA export. MET3 expression was then shut off by addition of excess methionine to the medium, and the progress of 
degradation of the MET3 mRNA trapped in the nucleus was assessed by Northern blot analysis. MET3 mRNA decay was not accelerated when we trapped Dcp2 in the nucleus by either using the edc $3 \Delta s c d 6 \Delta$ mutant or by overexpressing NLS ${ }^{S V 40}$-Dcp2, or NLS ${ }^{\mathrm{SV} 40-D c p 2}{ }^{\mathrm{CD}}$ as a control (Fig. 2G-I). Therefore, we conclude that Dcp2 is not actively decapping nuclear mRNAs for decay under these conditions. Taken together, our data do not support a nuclear function for Dcp2 and are consistent with the notion that the nuclear Dcp2 pool may serve as a buffer to control cytoplasmic decapping activity.

\section{Dcp1/Dcp2 performs essential functions on the cytoplasmic face of the ER}

If the nuclear pool of Dcp2 operates as a reservoir, Dcp2's essential function should be in the cytoplasm, in particular under stress conditions, when P-bodies are formed. Indeed, an edc3 $\Delta$ scd6 $\Delta$ mutant, in which Dcp2 becomes sequestered in the nucleus, showed impaired growth under stress (Fig. 3A). If this growth impairment was solely due to the Dcp2 localization, then Dcp2 overexpression (Fig. 3B and C) should rescue the growth defect. Overexpression of Dcp2 alleviated the growth phenotype of the edc3 $\Delta$ scd6 $\Delta$ mutant strain (Fig. 3D). These data indicate that Edc3 and Scd6 collaborate to regulate cytoplasmic Dcp2 levels and that excess Dcp2 can be stored in the nucleus until needed.

The simple overexpression, however, has two drawbacks. First, it increases the entire cytoplasmic pool of Dcp2 without providing spatial information and second the overexpressed protein can still be imported and trapped in the cell nucleus. To circumvent these potential pitfalls, we locked Dcp2 in the cytosol. To this end, we anchored Dcp2-GFP on the cytosolic face of the endoplasmic reticulum (ER) and of mitochondria (mito) by linking Dcp2-GFP to Dpm1 (Dcp2 ${ }^{\mathrm{ER}}$ ) or Fis1 (Dcp2 ${ }^{\text {mito }}$ ) (Fig. 3E and F). This approach allowed Dcp2 expression from its endogenous promoter and to be anchored at specific locations in the cytoplasm. We chose the ER because we have previously shown that P-bodies associate with ER membranes (Kilchert et al., 2010; Weidner et al., 2014; Wang et al., 2018), a result that was recently confirmed in mammalian cells (Lee et al., 2020). The mitochondria targeting was chosen as a control as we predicted based on our previously published results that mitochondrial Dcp2 should not be functional. The targeting approach worked as Dcp2 appended with Fis1 localized efficiently to mitochondria, and the construct carrying the Dpm1 colocalized with the ER. To ensure that the endogenous Dcp2 would not interfere with 
our assay, we acutely depleted endogenous Dcp2 using an auxin-inducible degron. This depletion worked efficiently as even under normal growth conditions Dcp2 depleted edc3s scd6s cells were unable to grow (Fig. 3G). Under these conditions, however, both mitochondrial and ER-localized Dcp2 rescued the growth phenotype. Under stress conditions, the ER-sequestered Dcp2 allowed much better survival when compared to Dcp2 on mitochondria (Fig. 3G). Therefore, ER-localized Dcp2 is chiefly responsible to cope with stress.

Nevertheless, we observed also some rescue by the mitochondria-localized Dcp2. To understand these results better, we first tested, whether P-bodies could be formed on mitochondria under stress conditions. Indeed, Dcp2 ${ }^{\text {mito }}$ formed foci, resembling P-bodies under glucose starvation (Fig. $3 \mathrm{H}$ ). These foci were also positive for two other bona fide P-body components, Dhh1 and Pat1 (Suppl. Fig. 3B and C), indicating that P-bodies can also form on mitochondria. Moreover, our data suggest that Dcp2 alone is sufficient to determine the location of P-body formation.

The ER and mitochondria are connected via contact sites to allow the exchange of lipids and ions (Elbaz and Schuldiner, 2011; Prinz, 2014). In yeast, the tethering complex ERMES stabilizes these contacts (Kornmann et al., 2009). We wondered whether the P-bodies formed containing Dcp2 ${ }^{\text {mito }}$ would be localized close to ERmitochondria contact sites. Indeed, P-bodies were detected next to or at the same site as the ERMES component Mdm34 (Fig. 3I and J). This presence at ER-mitochondria contact sites was essential for the ability of Dcp2 ${ }^{\text {mito }}$ to mount an appropriate stress response because destruction of the contacts by deleting the ERMES component Mmm1 abolished growth of Dcp2 ${ }^{\text {ito }}$ expressing cells under stress (Fig. 3K). Taken together, our data are consistent with the notion that functional P-body formation under stress takes place at the ER. Moreover, our data provide evidence that Dcp2 and Pbodies can also act in trans to cope with stress at ER-mitochondrial contact sites, albeit somewhat less efficiently.

\section{Scd6 and Edc3 bridge the interaction of Dcp2 with Dhh1 during P-body assembly}

Our data above suggest that Dcp2 localization determines where P-bodies assemble. Dcp2 can interact with both Scd6 and Edc3 (Fig. 4A), which in turn also interact with Dhh1, although their binding to Dhh1 was reported to be mutually exclusive (Fromm et al., 2012). Importantly, Dhh1 has been shown to drive phase separation, which is 
essential during P-body formation (Mugler et al., 2016; Hondele et al., 2019). First, we wanted to determine which parts of Scd6 and Edc3 are required to keep Dcp2 in the cytoplasm and to promote functional stress response. To this end, we carried out a domain analysis of Scd6 and Edc3 by overexpressing their individual domains alone or in combination in the edc3s scd6s background and determined the nuclearcytoplasmic distribution of Dcp2 and fitness at $37^{\circ} \mathrm{C}$ (Fig. 4B-E). As reported previously, overexpressing full-length of Scd6 impaired growth due to constitutive Pbody formation, even without stress (Nissan et al., 2010). Removal of the C-terminal region of Scd6 or Edc3 not only alleviated these growth defects, but restored growth to wild-type levels and reversed the P-body phenotype. Scd6 or Edc3 constructs lacking either the Dhh1 or the Dcp2 interaction site failed to restore growth and Dcp2 became enriched in the nucleus. Our data demonstrate that both Scd6 and Edc3 can bridge the interaction between Dcp2 and Dhh1, and that this connection is needed to keep Dcp2 from being transferred into the nucleus.

\section{Linking Dcp2 and Dhh1 drives P-body formation and functional stress response}

So far, our data suggest that Dcp2 and Dhh1 must come together to drive P-body formation under stress and that this process is mediated through interaction with either Scd6 or Edc3. To further corroborate our findings, we fused the N-terminal Dcp2 binding domain of Edc3 (Edc3(1-86), Edc $\left.3^{\mathrm{N}}\right)$ to Dhh1 and expressed the construct in the edc3 $\Delta s c d 6 \Delta$ Dcp2-GFP strain (Fig. 5A). Consistent with the data described above, Dcp2 is mostly nuclear in edc3 3 scd6 $6 \Delta$ cells (Fig. 5B). This localization did not change when either Dhh1 or the $E d c 3^{N}$ were expressed separately. However, the expression of the Edc $3^{\mathrm{N}}-\mathrm{Dhh} 1$ fusion protein sequestered Dcp2 in the cytoplasm. More importantly, when we repeated the experiment under glucose starvation, expression of only Edc3 ${ }^{\mathrm{N}}$-Dhh1 was sufficient to induce P-body formation (Fig. 5C and D). These P-bodies appear to be functional as they completely rescued the growth phenotype of edc3 3 scd6 $\Delta$ (Fig. 5E).

\section{Dcp2 is essential for P-body assembly}

Thus, our data suggest that Dcp2 is essential for P-body formation. However, it is assumed that P-body assembly is redundant and no single component is essential (Teixeira and Parker, 2007). Therefore, we decided to revisit the issue and determined 
P-body formation in our Dcp2-AID degron strain. Similarly, to what had been described previously, upon Dcp2 depletion, P-body formation was strongly impaired (Fig. 6A and B). Instead of 1-3 bright foci/cell, either only diffuse signal or multiple weak foci were observed, which were most conspicuous in the case of Edc3. To test whether these smaller foci might present smaller functional P-body units, we performed colocalization analyses. While Edc3 colocalized with Xrn1 and Pat1 very well in the presence of Dcp2, this level of co-localization dropped drastically in the absence of Dcp2 (Fig. 6C-E). Therefore, we conclude, that even though smaller speckles can be formed in the absence of Dcp2, they do not represent functional P-bodies, indicating that Dcp2 is essential for P-body formation. Moreover, our data are in accordance with previous data (Weidner et al., 2014) that granule formation and phase separation do not necessarily correlate with P-body functionality. 


\section{Discussion}

Even though P-bodies are an essential part of the cellular stress response, their assembly and cellular location are still debated and not fully understood. Recent studies highlighted the dynamics of individual P-body components during and the removal after stress (Xing et al., 2020) (Lee et al., 2020). The mechanism of their formation, however, is still not entirely clear. In another recent study, it was shown that the phase separation capability of the helicase Dhh1 contributed to P-body formation (Hondele et al., 2019). Yet, the dogma in the field is that no individual P-body component is essential for P-body formation. This reasoning is largely based on a study in which all major P-body components were individually deleted and the formation of foci with individual P-body members was analyzed (Teixeira and Parker, 2007) with a recent follow up from the same group (Rao and Parker, 2017). The initial systematic study showed that none of the deletions suppressed completely foci formation. Here, we show that even though some P-body components form speckles in the absence of Dcp2, these appear not to be functional P-bodies as they lack other P-body components. Moreover, our data provide strong evidence that functional Pbodies are formed at the ER under stress. We envisage an assembly pathway in which Dcp2 is associated with the ER through polysomes and/or other means. We have shown previously that a phosphorylated form of Dcp2 is enriched on ER-associated polysomes under normal growth conditions (Weidner et al., 2014). Moreover, immunoelectron microscopy showed P-bodies localizing in close proximity to the ER under stress (Kilchert et al., 2010; Weidner et al., 2014), confirmed by a recent live cell imaging approach in mammalian cells (Lee et al., 2020). Another P-body component, Scd6 is associated with polysomes in the cytoplasm and on ER membranes (Weidner et al., 2014), consistent with its role as a translational repressor. Upon stress, Scd6 would inhibit translation either through its direct interaction with elF4G, or with the help of Dhh1 which likewise has translational suppressor activity (Coller and Parker, 2005; Zeidan et al., 2018). However, in order to form a P-body, the local concentration of Scd6-Dhh1-mRNA complexes must be increased. This is achieved through binding to Dcp2 on the ER membrane (Fig. 7). We surmise that on the membrane, which reduces the diffusibility of the Dcp2-Scd6-Dhh1-mRNA complexes, it is much easier to gain a critical concentration to initiate Dhh1-driven phase separation. In a next step, Edc3, which like Scd6, can bind both Dcp2 and Dhh1 
is recruited. Because Edc3 is a dimer, it can act as a scaffold to further enhance recruitment of Dhh1 and P-body formation. In our model, the local concentration of Dcp2 on ER polysomes would be key to drive P-body formation. In support of our model, we find that first Dcp2 ${ }^{\mathrm{ER}}$ can rescue a $s c d 6 \Delta$ edc3 $\Delta$ mutant, while Dcp2 ${ }^{\text {mito }}$ cannot, unless concentrated at ER-mitochondrial contact sites. Second, directly linking Dcp2 to Dhh1 through the N-terminus of Edc3 (Edc3 $\left.{ }^{\mathrm{N}}-\mathrm{Dhh} 1\right)$ is sufficient for cells cope with stress. Moreover, an mRNA coupled to Scd6 is on the one hand translationally repressed in a Dhh1-dependent manner and on the other hand destabilized by Dcp2 (Zeidan et al. , 2018), supporting a temporal control in P-body assembly. In agreement with this notion, Scd6 and Edc3 interact with Dcp2 and Dhh1 in a mutually exclusive manner (Decker, Teixeira and Parker, 2007; Harigaya et al., 2010; Nissan et al., 2010; Fromm et al., 2012; Sharif et al., 2013). Finally, a recent finding did not even consider Scd6 an abundant P-body component after prolonged stress (Xing et al., 2020), again supporting the idea that Scd6 plays an important role early in P-body assembly and may then be displaced by Edc3 over time. Yet, Scd6 is a bona fide P-body component because tagged Dcp2 and Scd6 are about equally efficient to purify P-bodies and to determine the RNA content upon acute stress conditions (Weidner et al., 2014; Wang et al., 2018).

How does our model explain why deletions of for example PAT1 and LSM1 also affect P-body assembly? We propose that there is an initiation phase, and this is largely the process described above in which translational repression is intimately coupled to the initiation of P-body formation. In the next step, these molecular assemblies need to grow and to be stabilized, during which process those P-body components associated with 3' of the client mRNA are needed. In our view, in pat1 and Ism1 $1 \Delta$ the initial assembly of the 5' P-body members with the RNA at the ER is not defective, but rather stabilization of the assembly and its growth is disrupted. In this phase of $\mathrm{P}$-body stabilization, the numerous partially redundant interactions among different P-body members are important as previously described (Rao and Parker, 2017). Within 5 min of stress, such as glucose deprivation, P-bodies are formed. In particular under glucose starvation, initially multiple P-bodies become visible that apparently coalesce over $30 \mathrm{~min}$. Thus, P-bodies seem to mature over time, also perhaps to become more stable entities under non-adaptable stress conditions such as glucose starvation. In contrast, under adaptable stresses, this maturation process might be less critical, in particular if cells can reach a new 
equilibrium state within 30-45 $\mathrm{min}$ and start to dissociate P-bodies, as under hyperosmotic stress conditions (Kilchert et al., 2010).

If Dcp2 localization, and presumably activity, are critical for P-body formation, they should be strongly controlled. We know that the activity of Dcp2 can be regulated by phosphorylation and Edc3 (EDC4 in mammals) binding (Harigaya et al., 2010; Yoon, Choi and Parker, 2010; Chang et al., 2014; Paquette et al., 2018). Besides these onand off switches of activity, the cell might still want to control the protein localization as a second line to control activity. Upon stress, more Dcp2 would be needed immediately to cope with the remodeling of the proteome. Acute stress demands and acute response, in which a reserve or buffering pool would be advantageous. This pool may be hidden from the cytoplasm in order to prevent premature activation and to provide a more tuned response. Dcp2 can enter the nucleus through Kap95dependent import. The cellular/nuclear localization is determined by the presence of Scd6 and Edc3. Nuclear Dcp2 does not appear to be engaged in nuclear mRNA decay. However, it has been previously reported that nuclear Dcp2 can act as a transcriptional activator (Haimovich et al., 2013). Still, the essential function of Dcp2 appears to be in the cytoplasm where overexpression of catalytically dead Dcp2 was detrimental but it had no effect when locked in the nucleus. Dcp2 is not the only Pbody component that can be localized to the nucleus. The other, perhaps best documented component is Pat1 (Teixeira and Parker, 2007). Intriguingly, overexpression of Pat1 drives nuclear localization of Dhh1 (Sachdev et al., 2019). It appears as if nuclear-cytoplasmic distribution of key P-body components contribute to a built-in robustness to control cytoplasmic mRNA decay.

We propose that the arrangement of the mRNA decaying factors is orchestrated around Dcp2. Dcp2 serves as a platform for organizing the different elements of the mRNA machinery on a modular principle. It associates with Scd6 and Edc3, which contact the major regulator Dhh1 responsible for liquid-liquid phase separation. An ensemble of activators (Dcp1, Edc1, Edc2, Edc3, Pat1, Lsm1-7) acts concertedly to stimulate decapping. The downstream exonuclease Xrn1 recruited to the complex by Pat1 ensures the final processing of the transcript. Liquid-liquid phase separation and condensate formation can also take place in the absence of Dcp2. The lack of the decapping platform, however, leads to loss of spatial and temporal coordination of the mRNA decay factors and failure to properly organize in P-bodies under stress. 


\section{Material and Methods}

\section{Yeast Methods}

Strains used are listed in Supplementary Table 1. Standard genetic techniques were used throughout (Sherman, 1991). All modifications were carried out chromosomally, except where indicated. Chromosomal tagging and deletions were performed as described previously (Knop et al., 1999; Goldstein and McCusker, 1999; Gueldener et al., 2002; Janke et al., 2004). DCP2 and KAP95 were genomically tagged with an auxin-inducible degron $A I D *-9 M Y C$ and $A I D *-6 H A$ respectively using pNat-AID*-9myc and pHyg-AID*-6HA plasmid templates for generation of the C-terminal tagging cassettes (Morawska and Ulrich, 2013). NUP145 was truncated by genomic Cterminal tagging with $3 M Y C$ to yield the mutant nup145 $\triangle \mathrm{C}$ allele (nucleotides 1-1815 of the original ORF). TRP1 marker in the genomically integrated YIp204-ADH1p-AFB2 was disrupted using kanMX or hphMX4 cassettes.

\section{Plasmid construction}

Shuttle vectors for expression in yeast were prepared from $p R S 414-A D H$ and pRS415-GPD backbone plasmids (Mumberg, Müller and Funk, 1995) by Gibson assembly using NEBuilder HiFi DNA Assembly Cloning Kit according to the manifacturer's protocol (New England Biolabs). The fragments for the assembly were prepared by PCR using Q5 High Fidelity polymerase (New England Biolabs). The plasmids used along with details on their preparation are listed in Supplementary Table 2.

\section{Fluorescence Microscopy}

Yeast cells were cultured at $30^{\circ} \mathrm{C}$ in YPD in case of genomically integrated modifications or in the respective $\mathrm{HC}$-selection media when harboring plasmids. Cultures were diluted in HC-complete medium, re-grown to log phase, and either imaged directly or taken up in $\mathrm{HC}$-medium and subjected to stresses (medium without glucose for 30 min or medium supplemented with $0.2 \mathrm{M} \mathrm{CaCl}_{2}$ for 10 min). For Dcp2 depletion by auxin-inducible degron the logarithmically growing cells before treatment with stress or imaging were supplemented with $2 \mathrm{mM}$ auxin and cultured for $2 \mathrm{~h}$. Fluorescence and DIC images were acquired with an ORCA-flash 4.0 camera 
(Hamamatsu) mounted on an Axio Imager.M2 fluorescence microscope with a 63x Plan-Apochromat objective (Carl Zeiss, Germany) and a HXP $120 \mathrm{C}$ light source using ZEN 2.6 software. Image processing was performed using OMERO.insight client. For quantification of the number of foci the images from the same experiment were adjusted equally and inverted. A total of at least 300 cells from three independent experiments were quantified. Cell fluorescence measurements were carried out with ImageJ. For the nuclear-cytoplasmic GFP distribution the mean grey value of a region of interest (ROI) in the cell nucleus was normalized by the mean grey value of a ROI of the same size in the cytoplasm. A total of at least 75 cells from three independent experiments were quantified. The box and whiskers quantification graphs had the size of the box between the 25th and the 75th percentiles, and the whiskers - at the 5th and the 95th percentiles, the horizontal line marked the median and the cross indicated the mean value. The data sets were compared using a non-parametric test. $P$-values were indicated as follows: $0.1234-\mathrm{ns}, 0.0332-\left(^{\star}\right), 0.0021-\left(^{\star \star}\right), 0.0002-\left(^{\star \star \star}\right)$, $\left.<0.0001-{ }^{* \star \star *}\right)$. Colocalization was estimated using the JACoP plugin in ImageJ (Bolte and Cordelières, 2006). A total of at least 350 cells from three experiments were quantified.

\section{Total protein extracts and Western blot analysis}

For total protein extracts 5-7 ODs of cells were spun down, resuspended in $200 \mu \mathrm{l} 9$ M urea, $50 \mathrm{mM}$ Tris- $\mathrm{HCl}$, pH 8 with freshly added $0.5 \mathrm{mM}$ PMSF and beaten 2x20 s with $0.15 \mathrm{ml}$ glass beads $(0.25-0.5 \mathrm{~mm})$ at $6.5 \mathrm{~m} / \mathrm{s}$ at $4^{\circ} \mathrm{C} .2 x$ Laemmli buffer $(200 \mu \mathrm{l})$ was added and the samples were denatured at $65^{\circ} \mathrm{C}$ for 5 min. Samples for Western blot analysis were resolved on 10 or $12.5 \%$ SDS polyacrylamide gels and transferred on Amersham Protran Premium $0.45 \mu \mathrm{m}$ NC membrane. Membranes were decorated with the following antibodies: rabbit anti-GFP (Torrey Pines), mouse anti-Pgk1 (Invitrogen), mouse anti-Por1 (Invitrogen), and rabbit anti-Sec61 (generous gift from Martin Spiess, Biocenter Basel), goat anti-rabbit- and anti-mouse-HRPs were from Thermo. Membranes were developed with WesternBright ECL (Advansta) at a Fusion digital imager with Evolution-capt Edge software (Vilber, France). 


\section{Nuclear mRNA degradation assay}

The nuclear degradation of the MET3 mRNA was assayed in a nup145 $\Delta \mathrm{C}$ mutant background. nup $145 \Delta \mathrm{C}$ mutants are viable at $23^{\circ} \mathrm{C}$, but show a strong mRNA nuclear export defect at $37^{\circ} \mathrm{C}$ (Kufel et al., 2004). Logarithmically growing mutant cells were first shifted to a medium lacking methionine to induce the expression of the methionine-related genes $(4 \mathrm{~h})$, then to $37^{\circ} \mathrm{C}$ for $30 \mathrm{~min}$ to inhibit the nuclear mRNA export and finally methionine-related genes expression was shut off by addition of excess of methionine to the medium. Aliquots were taken out, spun down and frozen in liquid nitrogen at specific times. For preparation of total RNA the cell pellets were mixed with $300 \mu \mathrm{l} 50 \mathrm{mM}$ sodium acetate, pH 6, 10 mM EDTA, $25 \mu \mathrm{l} 20$ \% SDS and $300 \mu \mathrm{l}$ phenol-chloroform-iso-amyl alcohol, $\mathrm{pH} 4-5$. The mixtures were vortexed $30 \mathrm{~s}$ at top speed and incubated at $65^{\circ} \mathrm{C}$ for $6 \mathrm{~min}$. The samples were frozen in liquid nitrogen, left to thaw for $2 \mathrm{~min}$ at RT and spun at $20,000 \mathrm{xg}$ for $10 \mathrm{~min}, 4^{\circ} \mathrm{C}$. The aqueous layer was mixed with $200 \mu$ acidic phenol-chloroform-iso-amyl alcohol, vortexed at top speed for $30 \mathrm{~s}$ and spun again. To $180 \mu \mathrm{l}$ of the aqueous layer $20 \mu \mathrm{l} 3$ $\mathrm{M}$ sodium acetate and $600 \mu \mathrm{l}$ ethanol was added, the mixture was chilled at $-80^{\circ} \mathrm{C}$ for $2 \mathrm{~h}$ and centrifuged $\left(20,000 \mathrm{xg}, 30 \mathrm{~min}, 4^{\circ} \mathrm{C}\right)$. The pellets were washed with $400 \mu \mathrm{l} 75$ $\%$ ethanol, spun down again, air-dried at RT, and dissolved in $30 \mu \mathrm{l}$ water. An equal volume of 2X RNA Loading Dye (Thermo Fisher) was added, the samples were denatured at $65^{\circ} \mathrm{C}$ for 5 min and quickly chilled in an ice-water bath. For Northern blot analysis 3-5 $\mu$ g total RNA were separated on a $1.2 \%$ agarose gel containing formaldehyde. The RNA was transferred to a Hybond N+ membrane (Amersham) and hybridized to MET3 and SCR1 digoxigenin-labelled RNA probes. The probes were prepared by in vitro transcription with the MegaScript T7 kit (Ambion), Digoxigenin-11UTP (Roche) and purified DNA templates generated by PCR. One probe covering nucleobases 20-520 was used for ScR1 and two probes covering nucleobases 5-678 and 845-1446 were used in equimolar amounts for MET3. Membranes were decorated with sheep anti-digoxigenin Fab fragments coupled to peroxidase (Roche). The blots were developed with WesternBright ECL (Advansta) at a Fusion digital imager with Evolution-capt Edge software (Vilber, France). 


\section{Subcellular fractionation}

Logarithmically growing cells (25 ODs) were spun down, reduced in $100 \mathrm{mM}$ Tris- $\mathrm{HCl}$, $\mathrm{pH}$ 9.4, $10 \mathrm{mM}$ DTT for $10 \mathrm{~min}$ and washed with 2x5 ml spheroplast buffer $(0.7 \mathrm{M}$ sorbitol, 0.7xYPD, $50 \mathrm{mM}$ Tris- $\mathrm{HCl}, \mathrm{pH}$ 7.5). Cells were resuspended in spheroplast buffer (50 ODs/ml), $1 \mathrm{mM}$ DTT was added and the cell wall digested with Zymolyase $20 \mathrm{~T}$ (30 $\mu \mathrm{g} / \mathrm{OD})$ for $20 \mathrm{~min}$ at RT and gentle agitation. The spheroplast suspension was then layered on a 1-ml cushion of $7.5 \%$ Ficoll $400,0.7 \mathrm{M}$ sorbitol and spun at $1,000 \times$ for $2 \mathrm{~min}$. The spheroplast pellet was gently resuspended in $5 \mathrm{ml}$ spheroplast buffer and the spheroplasts were recovered for $1 \mathrm{~h}$ at RT and gentle rocking. The suspensions were spun down (1,000xg, $2 \mathrm{~min})$, the pellets were chilled on ice and washed with $0.8 \mathrm{ml}$ ice-cold lysis buffer (0.25 M sorbitol, $20 \mathrm{mM}$ HEPES, pH 6.8, 5 $\mathrm{mM} \mathrm{MgSO} 4,0.5 \mathrm{mM}$ PMSF). The pellets were resuspended in lysis buffer (100 ODs $/ \mathrm{ml}$ ), sonicated $2 \times 45 \mathrm{~s}$ in a low-output cleaning bath at $4^{\circ} \mathrm{C}$ and spun at 2,000xg for $2 \mathrm{~min}, 4^{\circ} \mathrm{C}$. Typically, $0.15 \mathrm{ml} \mathrm{S} 2$ was spun at $13,000 \times \mathrm{xg}$ for $10 \mathrm{~min}, 4^{\circ} \mathrm{C}$. The supernatant was spun at $110,000 \mathrm{xg}$ for $30 \mathrm{~min}, 4^{\circ} \mathrm{C}$ in a TLA 100.3 rotor (Beckman). $\mathrm{P} 13, \mathrm{P} 100$ and $\mathrm{S} 100$ were recovered, denatured with Laemmli buffer at $65^{\circ} \mathrm{C}$ for $5 \mathrm{~min}$ and analyzed by Western blotting.

\section{Acknowledgements}

We would like to thank Martin Spiess for the Sec61 antibody. We thank Rod Lim for suggesting Kap95 as responsible nuclear import factor for Dcp2. We acknowledge Maria Hondele and Ian G. Macara for critical comments on the manuscript. This work was supported by Swiss National Science Foundation (grants 310030B_163480 and 310030_185127) and the University of Basel.

Author contribution: KT and AS conceived the study and designed the experiments. $\mathrm{KT}$ performed the experiments. KT and AS interpreted the data. KT wrote the first draft. AS wrote the final draft with input from $\mathrm{KT}$.

\section{Competing interests}

The authors declare no competing interests. 


\section{References}

1. Bolte, S. and Cordelières, F. P. (2006) 'A guided tour into subcellular colocalization analysis in light microscopy', Journal of Microscopy. Blackwell Publishing Ltd, pp. 213-232. doi: 10.1111/j.1365-2818.2006.01706.x.

2. Chang, C. Te et al. (2014) 'The activation of the decapping enzyme DCP2 by DCP1 occurs on the EDC4 scaffold and involves a conserved loop in DCP1', Nucleic Acids Research, 42(8), pp. 5217-5233. doi: 10.1093/nar/gku129.

3. Coller, J. and Parker, R. (2005) 'General translational repression by activators of mRNA decapping', Cell, 122(6), pp. 875-886. doi:

10.1016/j.cell.2005.07.012.

4. Decker, C. J., Teixeira, D. and Parker, R. (2007) 'Edc3p and a glutamine/asparagine-rich domain of Lsm4p function in processing body assembly in Saccharomyces cerevisiae', Journal of Cell Biology, 179(3), pp. 437-449. doi: 10.1083/jcb.200704147.

5. Decourty, L. et al. (2008) 'Linking functionally related genes by sensitive and quantitative characterization of genetic interaction profiles', Proceedings of the National Academy of Sciences of the United States of America, 105(15), pp. 5821-5826. doi: 10.1073/pnas.0710533105.

6. Van Dijk, E. et al. (2002) 'Human Dcp2: A catalytically active mRNA decapping enzyme located in specific cytoplasmic structures', EMBO Journal, 21(24), pp. 6915-6924. doi: 10.1093/emboj/cdf678.

7. Elbaz, Y. and Schuldiner, M. (2011) 'Staying in touch: The molecular era of organelle contact sites', Trends in Biochemical Sciences. Trends Biochem Sci, pp. 616-623. doi: 10.1016/j.tibs.2011.08.004.

8. Fromm, S. A. et al. (2012) 'The structural basis of Edc3-and Scd6-mediated activation of the Dcp1:Dcp2 mRNA decapping complex', EMBO Journal, 31(2), pp. 279-290. doi: 10.1038/emboj.2011.408.

9. Goldstein, A. L. and McCusker, J. H. (1999) 'Three new dominant drug resistance cassettes for gene disruption in Saccharomyces cerevisiae', Yeast, 15(14), pp. 1541-1553. doi: 10.1002/(SICI)10970061(199910)15:14<1541::AID-YEA476>3.0.CO;2-K.

10. Gueldener, U. et al. (2002) 'A second set of loxP marker cassettes for Cremediated multiple gene knockouts in budding yeast', Nucleic acids research, 30(6), p. 23. doi: 10.1093/nar/30.6.e23.

11. Haimovich, G. et al. (2013) 'The fate of the messenger is pre-determined: A new model for regulation of gene expression', Biochimica et Biophysica Acta Gene Regulatory Mechanisms. NIH Public Access, pp. 643-653. doi: 10.1016/j.bbagrm.2013.01.004.

12. Harigaya, Y. et al. (2010) 'Identification and Analysis of the Interaction between Edc3 and Dcp2 in Saccharomyces cerevisiae', Molecular and Cellular Biology, 30(6), pp. 1446-1456. doi: 10.1128/mcb.01305-09.

13. Hondele, M. et al. (2019) 'DEAD-box ATPases are global regulators of phaseseparated organelles', Nature, 573(7772), pp. 144-148. doi: 10.1038/s41586019-1502-y.

14. Janke, C. et al. (2004) 'A versatile toolbox for PCR-based tagging of yeast genes: New fluorescent proteins, more markers and promoter substitution cassettes', Yeast, 21(11), pp. 947-962. doi: 10.1002/yea.1142.

15. Kilchert, C. et al. (2010) 'Defects in the secretory pathway and high Ca2+ induce multiple P-bodies', Molecular Biology of the Cell, 21(15), pp. 2624- 
2638. doi: 10.1091/mbc.E10-02-0099.

16. Kilchert, C. (2010) 'mRNA localization and turnover in mutants of the small GTPase Arf1p of Saccharomyces cerevisiae', Doctoral thesis, University of Basel.

17.Knop, M. et al. (1999) 'Epitope tagging of yeast genes using a PCR-based strategy: More tags and improved practical routines', Yeast, 15(10 B), pp. 963-972. doi: 10.1002/(SICI)1097-0061(199907)15:10B<963::AIDYEA399>3.0.CO;2-W.

18. Kornmann, B. et al. (2009) 'An ER-mitochondria tethering complex revealed by a synthetic biology screen', Science, 325(5939), pp. 477-481. doi: 10.1126/science.1175088.

19. Kosugi, S. et al. (2009) 'Systematic identification of cell cycle-dependent yeast nucleocytoplasmic shuttling proteins by prediction of composite motifs', Proceedings of the National Academy of Sciences of the United States of America, 106(25), pp. 10171-10176. doi: 10.1073/pnas.0900604106.

20. Kshirsagar, M. and Parker, R. (2004) 'Identification of Edc3p as an Enhancer of mRNA Decapping in Saccharomyces cerevisiae', Genetics, 166(2), pp. 729-739. doi: 10.1534/genetics.166.2.729.

21. Kufel, J. et al. (2004) 'Nuclear Pre-mRNA Decapping and 5' Degradation in Yeast Require the Lsm2-8p Complex', Molecular and Cellular Biology, 24(21), pp. 9646-9657. doi: 10.1128/mcb.24.21.9646-9657.2004.

22. Lee, J. E. et al. (2020) 'Endoplasmic reticulum contact sites regulate the dynamics of membraneless organelles', Science, 367(6477). doi: 10.1126/science.aay7108.

23. Luo, Y. et al. (2020) 'Global Profiling of Cellular Substrates of Human Dcp2', Biochemistry. doi: 10.1021/acs.biochem.0c00069.

24. Morawska, M. and Ulrich, H. D. (2013) 'An expanded tool kit for the auxininducible degron system in budding yeast', Yeast, 30(9), pp. 341-351. doi: 10.1002/yea.2967.

25. Mugler, C. F. et al. (2016) 'ATPase activity of the DEAD-box protein Dhh1 controls processing body formation', eLife, 5(OCTOBER2016). doi: 10.7554/eLife.18746.

26. Mumberg, D., Müller, R. and Funk, M. (1995) 'Yeast vectors for the controlled expression of heterologous proteins in different genetic backgrounds', Gene, 156(1), pp. 119-122. doi: 10.1016/0378-1119(95)00037-7.

27. Nguyen Ba, A. N. et al. (2009) 'NLStradamus: A simple Hidden Markov Model for nuclear localization signal prediction', BMC Bioinformatics, 10(1), p. 202. doi: 10.1186/1471-2105-10-202.

28. Nissan, T. et al. (2010) 'Decapping Activators in Saccharomyces cerevisiae Act by Multiple Mechanisms', Molecular Cell, 39(5), pp. 773-783. doi: 10.1016/j.molcel.2010.08.025.

29. Paquette, D. R. et al. (2018) 'Control of mRNA decapping by autoinhibition', Nucleic Acids Research, 46(12), pp. 6318-6329. doi: 10.1093/nar/gky233.

30. Parker, R. (2012) 'RNA degradation in Saccharomyces cerevisae', Genetics, 191(3), pp. 671-702. doi: 10.1534/genetics.111.137265.

31. Prinz, W. A. (2014) 'Bridging the gap: Membrane contact sites in signaling, metabolism, and organelle dynamics', Journal of Cell Biology. Rockefeller University Press, pp. 759-769. doi: 10.1083/jcb.201401126.

32. Rajyaguru, P., She, M. and Parker, R. (2012) 'Scd6 Targets elF4G to Repress Translation: RGG Motif Proteins as a Class of elF4G-Binding Proteins', 
Molecular Cell, 45(2), pp. 244-254. doi: 10.1016/j.molcel.2011.11.026.

33. Ramachandran, V., Shah, K. H. and Herman, P. K. (2011) 'The cAMPDependent Protein Kinase Signaling Pathway Is a Key Regulator of P Body Foci Formation', Molecular Cell, 43(6), pp. 973-981. doi: 10.1016/j.molcel.2011.06.032.

34. Rao, B. S. and Parker, R. (2017) 'Numerous interactions act redundantly to assemble a tunable size of $\mathrm{P}$ bodies in Saccharomyces cerevisiae', Proceedings of the National Academy of Sciences of the United States of America, 114(45), pp. E9569-E9578. doi: 10.1073/pnas.1712396114.

35. Sachdev, R. et al. (2019) 'Pat1 promotes processing body assembly by enhancing the phase separation of the DEAD-box ATPase Dhh1 and RNA', eLife, 8. doi: 10.7554/eLife.41415.

36. Sharif, H. et al. (2013) 'Structural analysis of the yeast Dhh1-Pat1 complex reveals how Dhh1 engages Pat1, Edc3 and RNA in mutually exclusive interactions', Nucleic Acids Research, 41(17), pp. 8377-8390. doi: 10.1093/nar/gkt600.

37. Sherman, F. (1991) 'Getting Started with Yeast', Methods in Enzymology, 194(C), pp. 3-21. doi: 10.1016/0076-6879(91)94004-V.

38. Sheth, U. and Parker, R. (2003) 'Decapping and decay of messenger RNA occur in cytoplasmic processing bodies', Science, 300(5620), pp. 805-808. doi: $10.1126 /$ science.1082320.

39. Sikorski, R. S. and Hieter, P. (1989) 'A system of shuttle vectors and yeast host strains designed for efficient manipulation of DNA in Saccharomyces cerevisiae.', Genetics, 122(1), pp. 19-27. Available at: /pmc/articles/PMC1203683/?report=abstract (Accessed: 31 August 2020).

40. Teixeira, D. and Parker, R. (2007) 'Analysis of P-body assembly in Saccharomyces cerevisiae', Molecular Biology of the Cell, 18(6), pp. 22742287. doi: 10.1091/mbc.E07-03-0199.

41. Tesina, P. et al. (2019) 'Structure of the 80 S ribosome-Xrn1 nuclease complex', Nature Structural and Molecular Biology, 26(4), pp. 275-280. doi: 10.1038/s41594-019-0202-5.

42. Vindry, C. et al. (2017) 'Dual RNA Processing Roles of Pat1b via Cytoplasmic Lsm1-7 and Nuclear Lsm2-8 Complexes', Cell Reports, 20(5), pp. 1187-1200. doi: 10.1016/j.celrep.2017.06.091.

43. Wang, C. et al. (2018) 'Context-dependent deposition and regulation of mRNAs in P-bodies', eLife, 7. doi: 10.7554/eLife.29815.

44. Weidner, J. et al. (2014) 'The polysome-associated proteins Scp160 and Bfr1 prevent $\mathrm{P}$ body formation under normal growth conditions', Journal of Cell Science, 127(9), pp. 1992-2004. doi: 10.1242/jcs.142083.

45. Xing, W. et al. (2020) 'A quantitative inventory of yeast $P$ body proteins reveals principles of composition and specificity', eLife, 9, pp. 1-63. doi: 10.7554/eLife.56525.

46. Yoon, J. H., Choi, E. J. and Parker, R. (2010) 'Dcp2 phosphorylation by Ste20 modulates stress granule assembly and mRNA decay in Saccharomyces cerevisiae', Journal of Cell Biology, 189(5), pp. 813-827. doi: 10.1083/jcb.200912019.

47. Zeidan, Q. et al. (2018) 'Conserved mRNA-granule component Scd6 targets Dhh1 to repress translation initiation and activates Dcp2-mediated mRNA decay in vivo', PLoS Genetics, 14(12). doi: 10.1371/journal.pgen.1007806. 


\section{Figure 1}

A

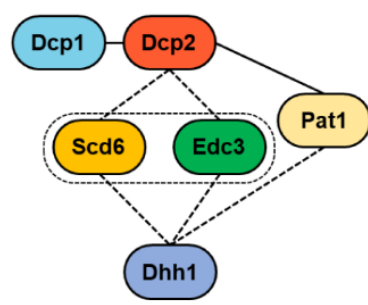

S. cerevisiae

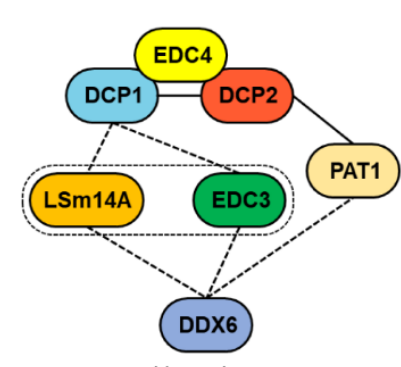

H. sapiens
B

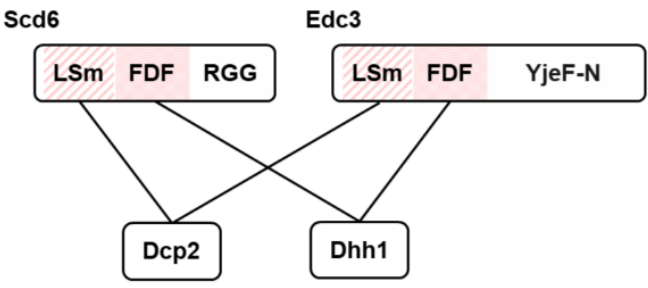

C

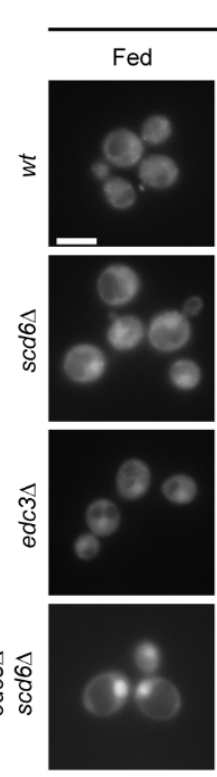

Dcp2-GFP
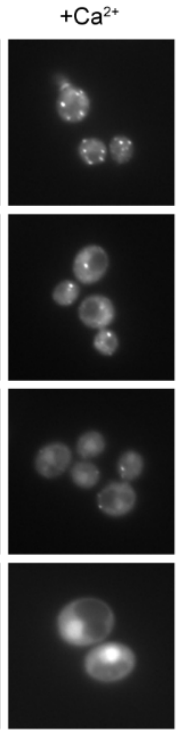

D

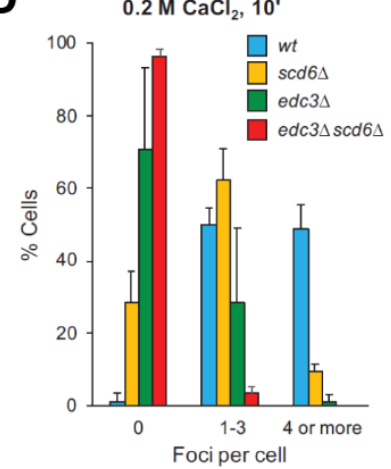

E

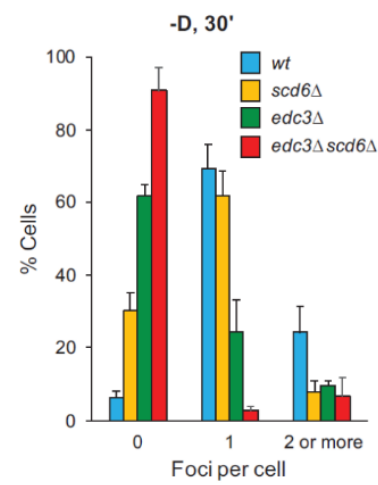

F

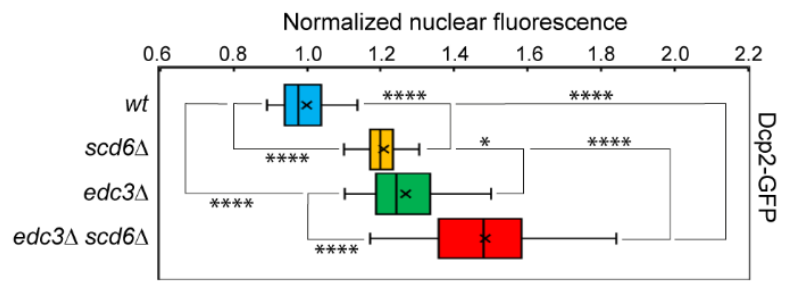

G

Nup84-

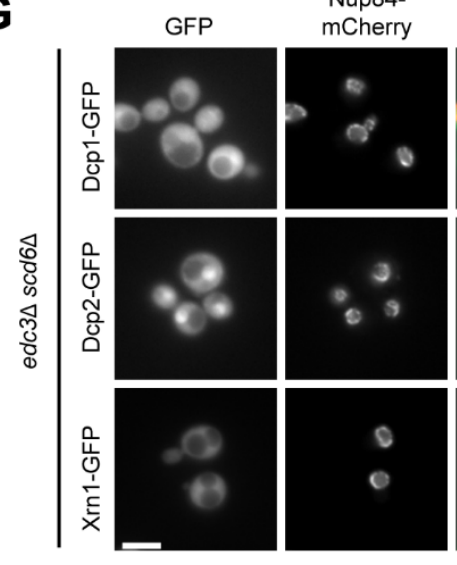

Merge

H

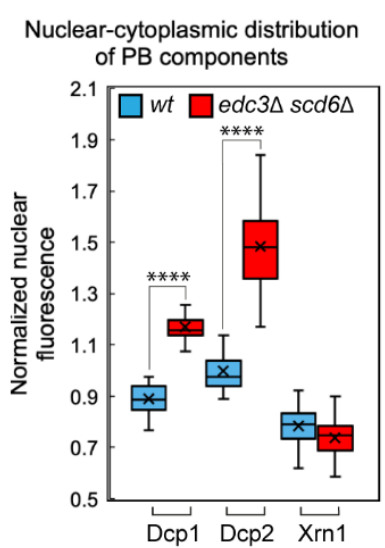

edc $3 \Delta s c d 6 \Delta$
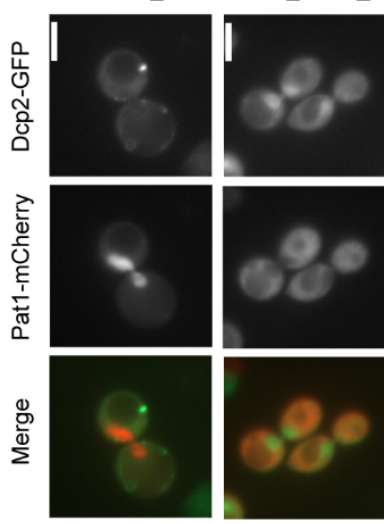
Figure 1. Concomitant loss of Scd6 and Edc3 blocks P-body assembly and drives nuclear accumulation of Dcp2. (A) Schematic representation of the evolutionary conserved basic PB-components in budding yeast and humans. Dashed lines indicate mutually exclusive interactions. (B) Both Scd6 and Edc3 ensure interaction between Dcp2 and Dhh1 through common structural motifs. (C) Loss of function of SCD6 and EDC3 leads to defects in PB formation under stress and nuclear enrichment of Dcp2. Logarithmically growing cells expressing genomically tagged Dcp2-GFP were imaged either directly or first shifted to the respective stress conditions (0.2 $\mathrm{M} \mathrm{CaCl}_{2}$ for $10 \mathrm{~min}$ or glucose deprivation for $\left.30 \mathrm{~min}\right)$. Scale bar $5 \mu \mathrm{m}$. (D) and (E) Quantification of the number of GFP foci of the data in panel C from at least 3 independent experiments. (F) Quantification of the nuclear-cytoplasmic Dcp2GFP distribution of the data panel $C$ from at least 3 independent experiments. The mean nuclear GFP fluorescence of a small area of the cell nucleus was normalized to the mean GFP fluorescence of small area of the cytoplasm of the same cell. $(G)$ In edc3 $\Delta s c d 6 \Delta$ the decapping complex components Dcp1 and Dcp2 are enriched in the cell nucleus while the exonuclease Xrn1 is not. Logarithmically growing edc $3 \Delta s c d 6 \Delta$ cells expressing genomically tagged Dcp1-, Dcp2- or Xrn1-GFP and the nuclear marker Nup84-mCherry were imaged without additional treatment. Scale bar $5 \mu \mathrm{m}$. (H) Quantification of the nuclear-cytoplasmic distribution of the GFP-tagged proteins in panel H. (I) Dcp2 and Pat1 are imported into the cell nucleus through different mechanisms. Logarithmically growing cells expressing Dcp2-GFP from a genomic locus and Pat1-mCherry from a low-copy plasmid on its own promoter were imaged without additional treatment. Scale bar $5 \mu \mathrm{m}$. 
bioRxiv preprint doi: https://doi.org/10.1101/2020.09.07.285700; this version posted September 8, 2020. The copyright holder for this preprint (which was not certified by peer review) is the author/funder, who has granted bioRxiv a license to display the preprint in perpetuity. It is made available under aCC-BY 4.0 International license.

\section{Figure 2}

A

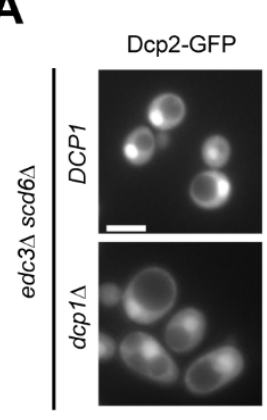

D

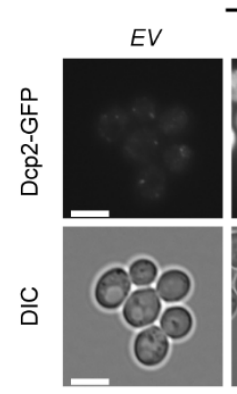

G

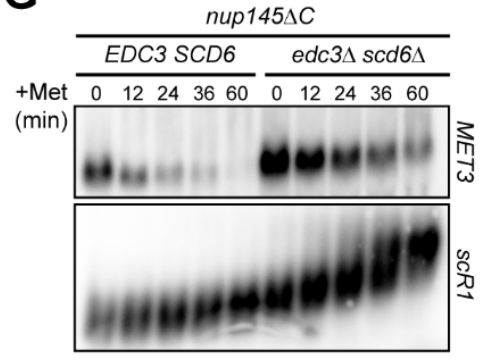

B

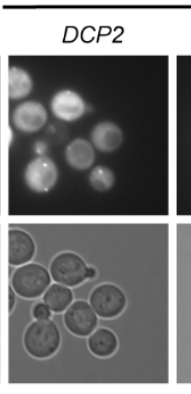

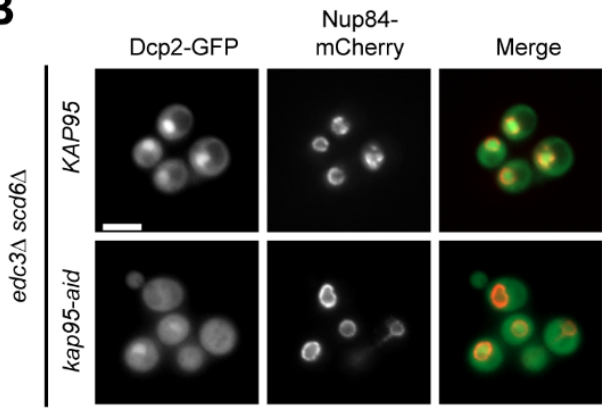

GPDp-DCP2-GFP

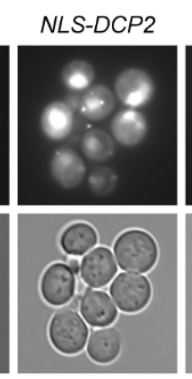

H

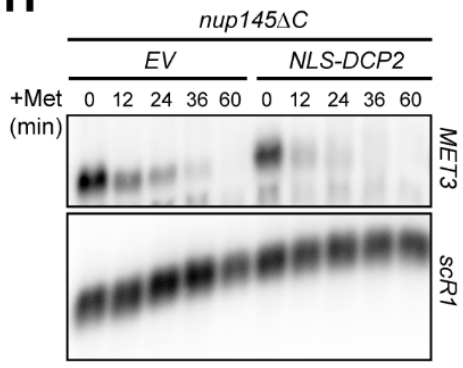

C

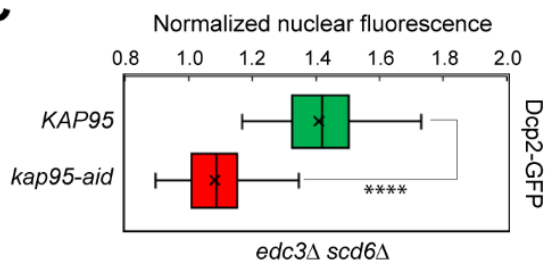

E

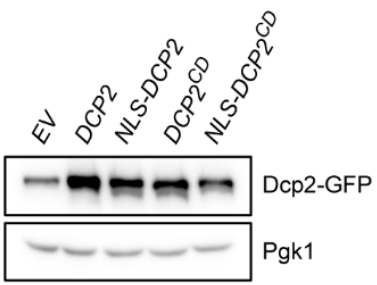

F

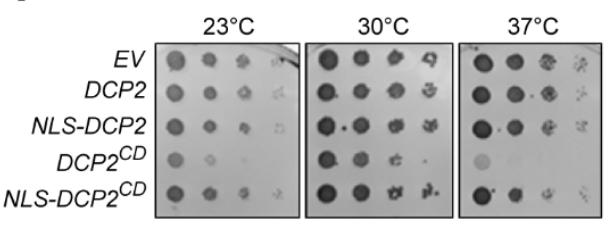

I

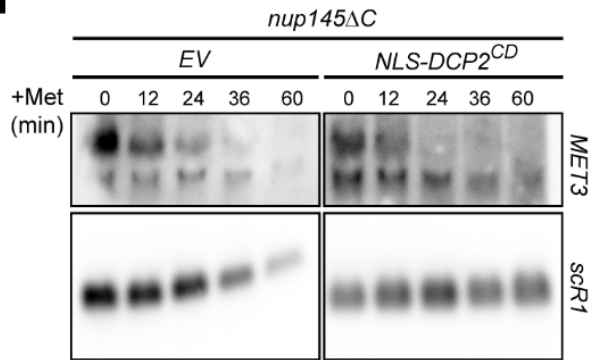


Figure 2. The decapping complex is stored in the nucleus as a readily releasable pool. (A) Dcp1 is not required for nuclear enrichment of Dcp2 in edc3 $s c d 6 \Delta$. Logarithmically growing cells expressing genomically tagged Dcp2-GFP were imaged without further treatment. Scale bar $5 \mu \mathrm{m}$. (B) Dcp2 nuclear import is dependent on Kap95. KAP95 in edc3 3 scd6 $\Delta$ cells expressing genomically tagged Dcp2-GFP and the nuclear marker Nup84-mCherry was C-terminally tagged with an auxin-inducible degron. Logarithmically growing cells from the starting strain and the KAP95-tagged variant were imaged without further treatment. Scale bar $5 \mu \mathrm{m}$. (C) Quantification of the nuclear-cytoplasmic Dcp2-GFP distribution of the data in panel B. (D) and (E) Overexpression of NLS-appended and catalytically dead (CD) variants of Dcp2. Cells expressing Dcp2-GFP from a genomic locus were transformed with a low-copy plasmid expressing different versions of Dcp2-GFP from the strong GPD promoter. Cells from a logarithmically grown culture were imaged by fluorescence microscopy (D, scale bar $5 \mu \mathrm{m}$ ) or GFP expression was analyzed by Western blotting (F). High levels of Dcp2 as well as Dcp2 sequestered in the cell nucleus do not affect cell fitness. Serial dilutions of the strains in panels (D) and (E) were spotted on YPD-agar, and incubated for 2 days at the indicated temperatures. $(\mathrm{G}),(\mathrm{H})$ and $(\mathrm{I})$ Northern blot analysis of MET3 mRNA nuclear degradation. nup145 $\triangle \mathrm{C}$ strain background was either deleted for EDC3 and SCD6, or transformed with low-copy plasmids expressing the indicated Dcp2 constructs. Logarithmically growing cultures were starved for methionine to induce the expression of $M E T 3$ and then shifted to $37^{\circ} \mathrm{C}$ to inhibit mRNA nuclear export. MET3 transcription was then blocked by addition of methionine and aliquots for Northern blotting analysis were taken at the indicated time points. The scR1 mRNA served as a loading control. 
bioRxiv preprint doi: https://doi.org/10.1101/2020.09.07.285700; this version posted September 8, 2020. The copyright holder for this preprint (which was not certified by peer review) is the author/funder, who has granted bioRxiv a license to display the preprint in perpetuity. It is made available under aCC-BY 4.0 International license.

\section{Figure 3}

A

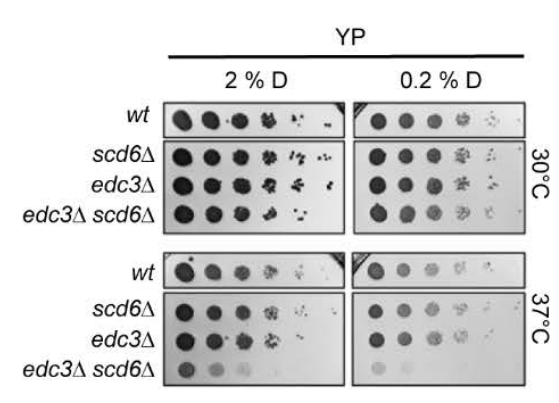

B

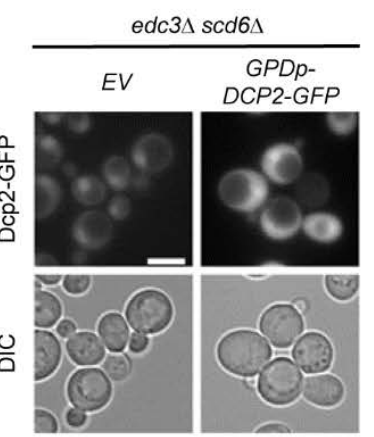

C

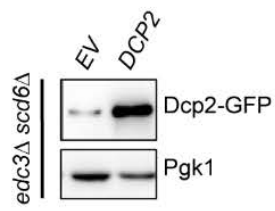

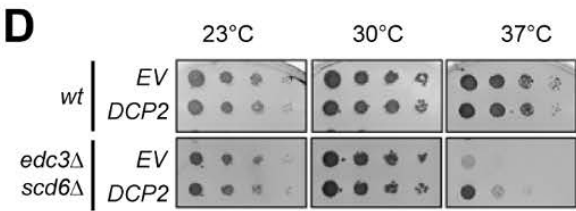

E

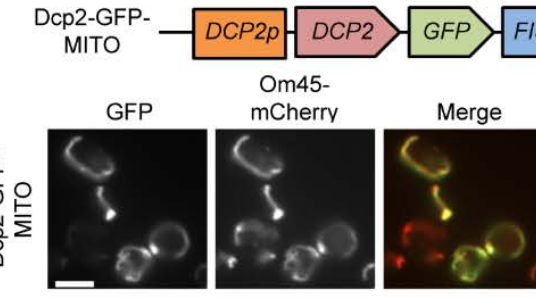

FIS1 - $\quad$ F $\underset{\text { ER }}{\text { Dcp2-GFP }}$

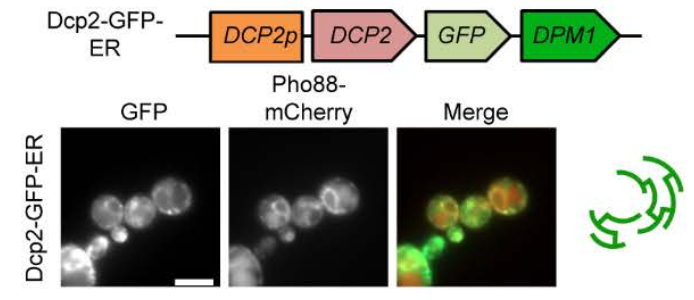

G

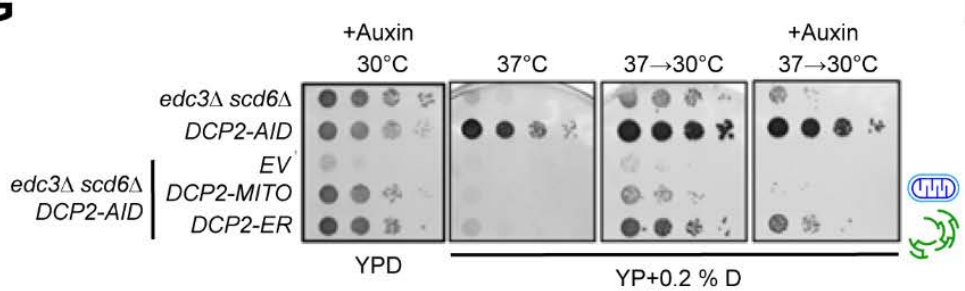

H
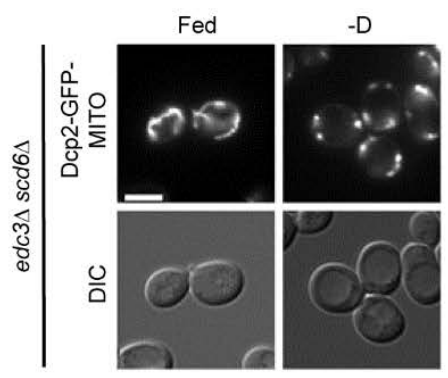

I
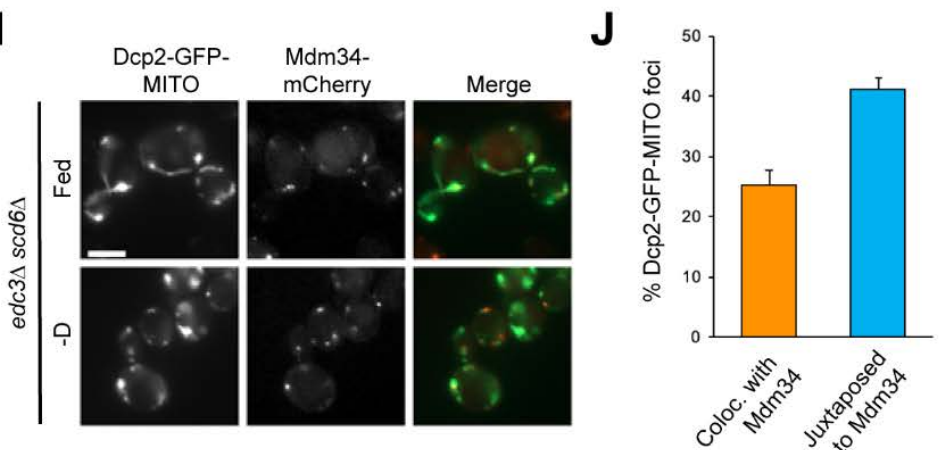

K

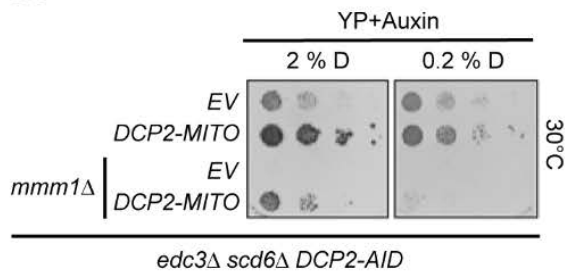


Figure 3. Dcp1/Dcp2 performs essential functions on the cytoplasmic face of the ER. (A) edc3 $\Delta s c d 6 \Delta$ displays impaired growth under stress. Serial dilutions of the indicated strains were spotted on YP-agar containing 2 or $0.2 \%$ glucose, and incubated at $30^{\circ} \mathrm{C}$ or $37^{\circ} \mathrm{C}$ for 2 days. (B) and (C) Dcp2 overexpression in edc3s scd6 6 . Dcp2-GFP was expressed on a GPD promoter from a low-copy plasmid on top of genomically tagged Dcp2-GFP in edc3 $s c d 6 \Delta$. Logarithmically growing cells were either imaged directly (B, scale bar $5 \mu \mathrm{m})$ or GFP expression was analyzed by Western blotting (C). (D) Overexpression of Dcp2 partially rescues growth of edc3 $s c d 6 \Delta$ under stress. Wild-type and edc3 $s c d 6 \Delta$ strains expressing Dcp2-GFP on a GPD promoter from a low-copy plasmid were serially diluted and spotted on YPD-agar, and incubated at the indicated temperatures for 2 days. (E) Structure and subcellular localization of the Dcp2-GFP-MITO. edc3 $\Delta s c d 6 \Delta$ with genomically tagged Om45mCherry were transformed with a low-copy plasmid expressing Dcp2-GFP-Fis1 from a $D C P 2$ promoter. Logarithmically growing cultures were imaged directly, scale bar 5 $\mu \mathrm{m}$. (F) Structure and subcellular localization of the Dcp2-GFP-ER. edc $3 \Delta s c d 6 \Delta$ cells with genomically tagged Pho88-mCherry were transformed with a low-copy plasmid expressing Dcp2-GFP-Dpm1 from a DCP2 promoter. Logarithmically growing cultures were imaged directly, scale bar $5 \mu \mathrm{m}$. (G) Dcp2 at the ER is required for growth under stress. edc3 $\triangle s c d 6 \triangle$ with $D C P 2$ tagged genomically with an auxin-inducible degron were transformed with low-copy plasmids expressing the indicated Dcp2-constructs from the endogenous $D C P 2$ promoter. Serial dilutions of the respective logarithmically growing cultures were spotted on YP-agar with 2 or $0.2 \%$ glucose, supplemented with $0.2 \mathrm{M}$ auxin as indicated. Agar plates were incubated either for 2 days at $30^{\circ} \mathrm{C}$ or first for 2 days at $37^{\circ} \mathrm{C}$, and then for 3 days at $30^{\circ} \mathrm{C}$. (H) Dcp2-MITO forms foci upon glucose starvation. edc3 $3 s c d 6 \Delta$ strain with the endogenous $D C P 2$ tagged with an auxin-inducible degron was transformed with a low-copy plasmid expressing the Dcp2-MITO construct from a DCP2 promoter. Logarithmically growing cultures were treated with $2 \mathrm{mM}$ auxin for $2 \mathrm{~h}$, and either imaged directly or upon 30 min glucose deprivation. Scale bar $5 \mu \mathrm{m}$. (I) Dcp2-MITO foci localize in close vicinity of ERmitochondria contact sites. edc3 $\Delta s c d 6 \Delta$ strain with genomically tagged Mdm34mCherry and Dcp2-Aid was transformed with a low-copy Dcp2-GFP-MITO construct. Logarithmically growing cells were treated as for panel $3 \mathrm{H}$. (J) Quantification of the number of GFP foci in panel 3-I colocalizing or juxtaposed to Mdm34 upon 30 min of glucose starvation. (K) ER-Mitochondria contact sites are essential for the functioning of Dcp2-MITO under stress. edc3 $3 s c d 6 \Delta$ with the endogenous DCP2 tagged with an auxin-inducible degron was deleted for $M M M 1$ and transformed with a low-copy plasmid expressing Dcp2-MITO from a DCP2 promoter. Logarithmically growing cultures of the indicated strains were serially diluted and spotted on YP-agar with 2 or $0.2 \%$ glucose, supplemented with $0.2 \mathrm{M}$ auxin, and incubated at $30^{\circ} \mathrm{C}$ for 3 days. 
bioRxiv preprint doi: https://doi.org/10.1101/2020.09.07.285700; this version posted September 8, 2020. The copyright holder for this preprint (which was not certified by peer review) is the author/funder, who has granted bioRxiv a license to display the preprint in perpetuity. It is made available under aCC-BY 4.0 International license.

Figure 4

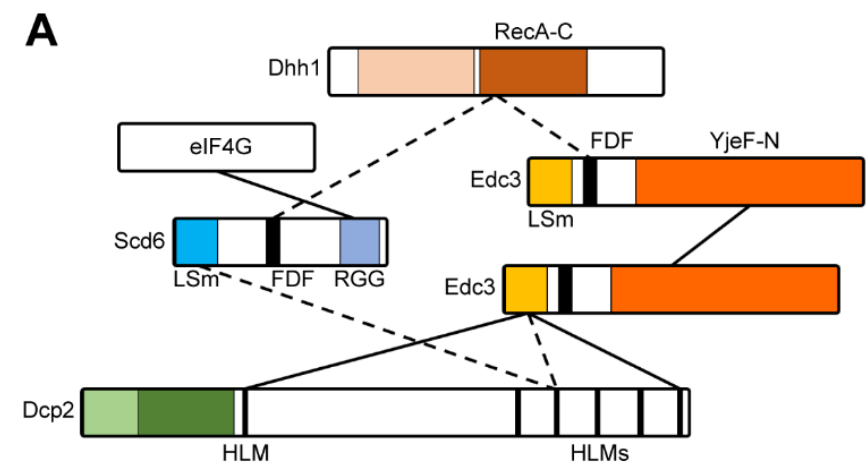

B

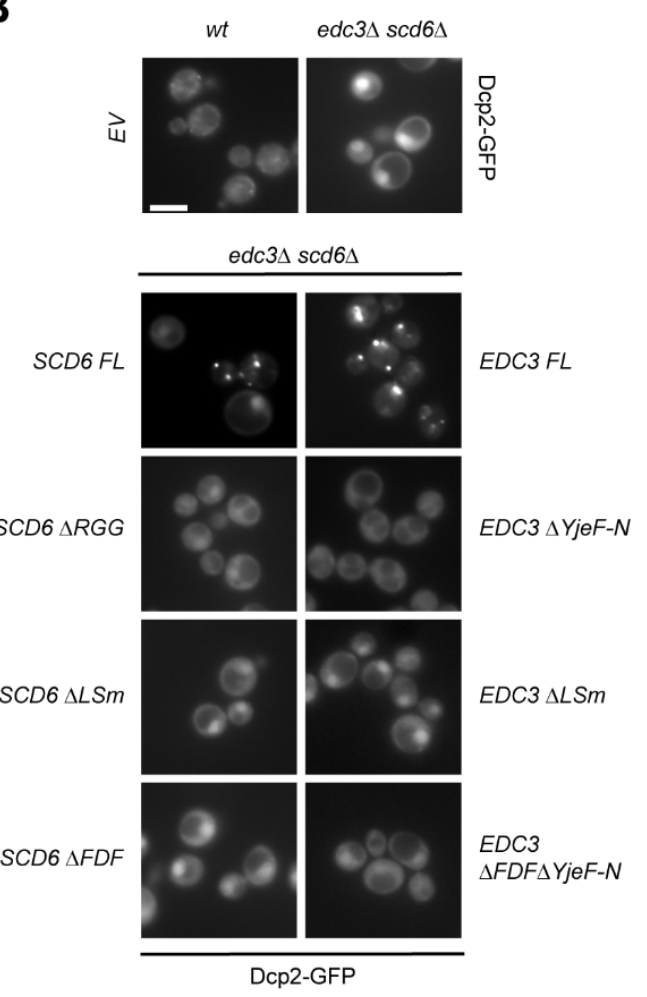

D

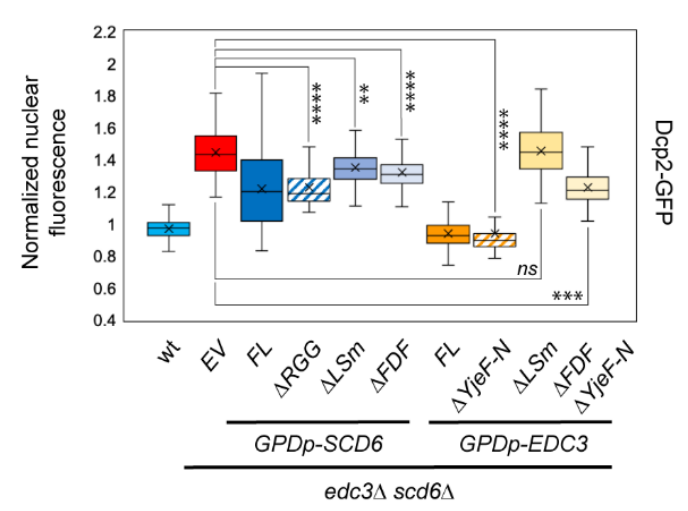

E edc3 $\Delta s c d 6 \Delta$ constructs pCEN-GPDp-EDC3constructs

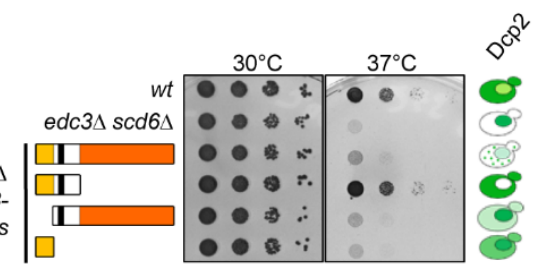


Figure 4. Scd6 and Edc3 bridge the interaction of Dcp2 with Dhh1 during P-body assembly. (A) Schematic representation of the domain structure and interactions of Scd6, Edc3, Dcp2 and Dhh1. Dashed lines represent mutually exclusive interactions. (B) Bridging Dcp2 to Dhh1 is required to keep Dcp2 in the cytoplasm. Wild-type or edc3 $\Delta$ scd6 $\Delta$ with genomically tagged Dcp2-GFP were transformed with low-copy plasmids expressing the indicated SCD6 or EDC3 constructs from the strong GPD promoter. Logarithmically growing cells were imaged without further treatment. Scale bar $5 \mu \mathrm{m}$. (C) Quantification of the nuclear-cytoplasmic GFP distribution in the cells on panel 4-B. D and E. Scd6 and Edc3 bridge Dcp2 to Dhh1 to cope with stress. Logarithmically growing cultures from the strains in panels $B$ and $C$ were serially diluted and spotted on HC-agar lacking leucine, and incubated at 30 or $37^{\circ} \mathrm{C}$ for 2 days. The cartoon shows the Dcp2-GFP nuclear-cytoplasmic distribution. 
bioRxiv preprint doi: https://doi.org/10.1101/2020.09.07.285700; this version posted September 8, 2020. The copyright holder for this preprint (which was not certified by peer review) is the author/funder, who has granted bioRxiv a license to display the preprint in perpetuity. It is made available under aCC-BY 4.0 International license.

\section{Figure 5}

A

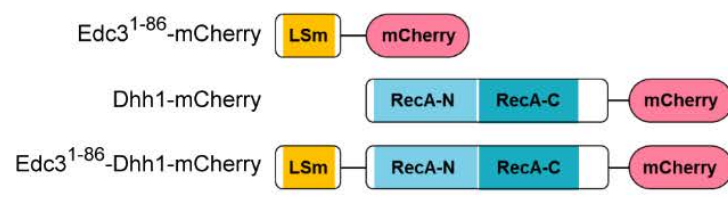

C

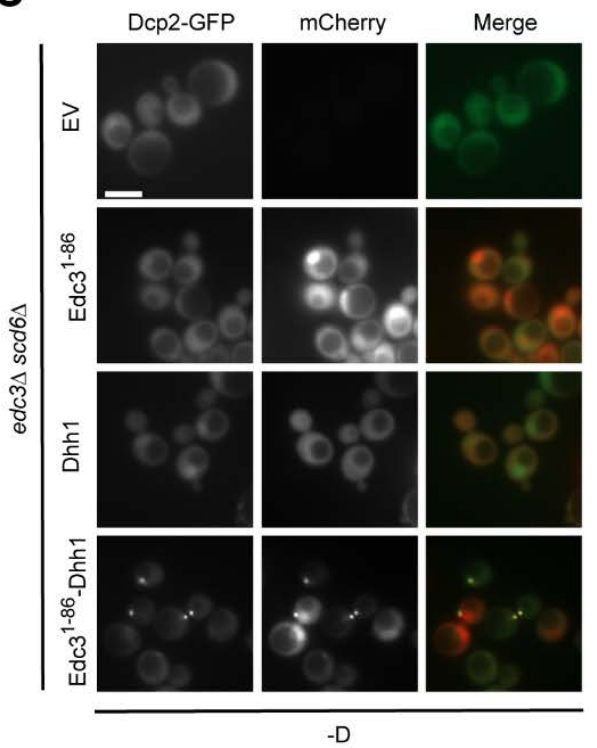

B

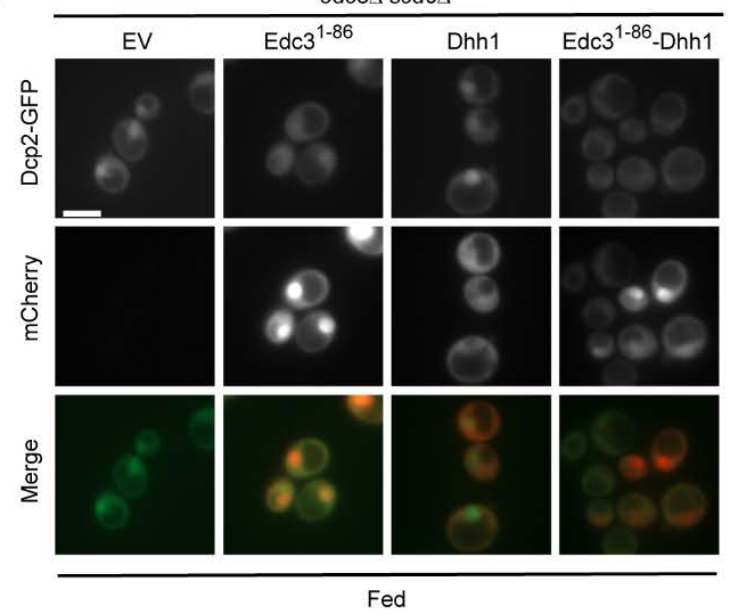

E

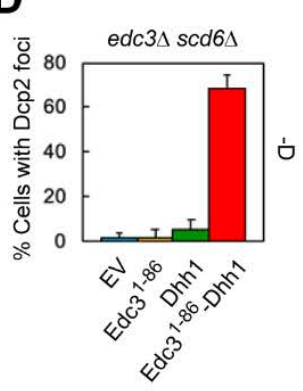

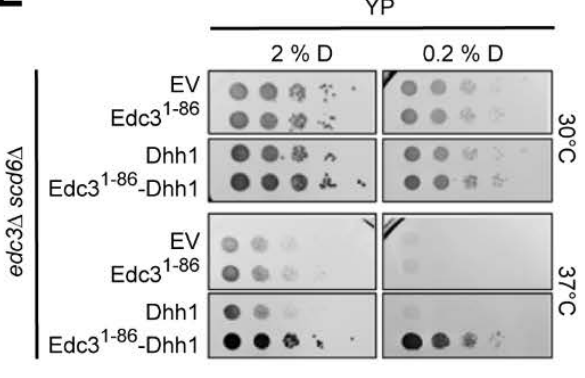


Figure 5. Linking Dcp2 and Dhh1 drives P-body formation and functional stress response. (A) Structure of the Edc3 $3^{1-86}$-Dhh1-mCherry fusion and the control Edc3 ${ }^{1-}$ ${ }^{86}$-mCherry and Dhh1-mCherry constructs. All construct were expressed from a lowcopy plasmid on the endogenous $D H H 1$ promoter. B-D. Linking Dhh1 to Dcp2 rescues Dcp2's cytoplasmic localization and PB formation in edc3 $\Delta$ scd6 $\Delta$. edc3 $\Delta$ scd6 $\Delta$ with genomically tagged Dcp2-GFP was transformed with the constructs from panel 5A. Logarithmically growing cells were imaged either directly (B) or after 30 min of glucose deprivation (C). Scale bar $5 \mu \mathrm{m}$. (D) Quantification of the number of cells forming GFP foci upon $30 \mathrm{~min}-\mathrm{D}$. (E) The Dcp2-Dhh1 interaction insures cell fitness under increased stress. Serial dilutions of the strains from panel B and C were spotted on YP-agar with 2 or $0.2 \%$ glucose, and incubated at the indicated temperatures for 2 days. 
bioRxiv preprint doi: https://doi.org/10.1101/2020.09.07.285700; this version posted September 8, 2020. The copyright holder for this

preprint (which was not certified by peer review) is the author/funder, who has granted bioRxiv a license to display the preprint in perpetuity. It is made available under aCC-BY 4.0 International license.

\section{Figure 6}

A
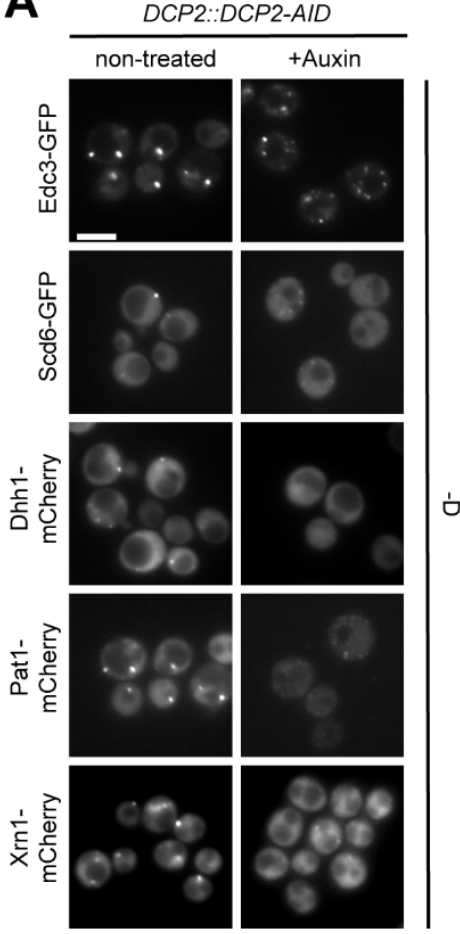

C

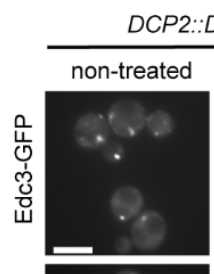

DCP2::DCP2-AID
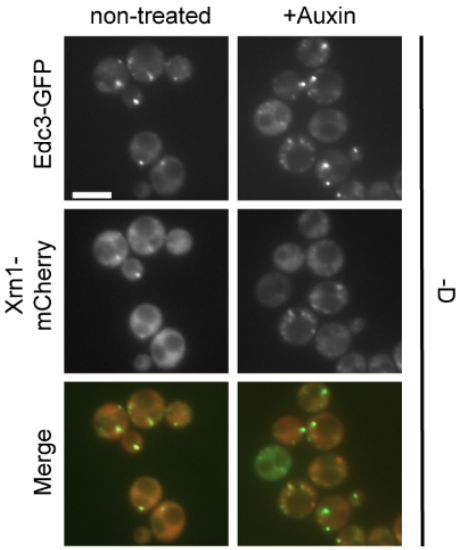

B
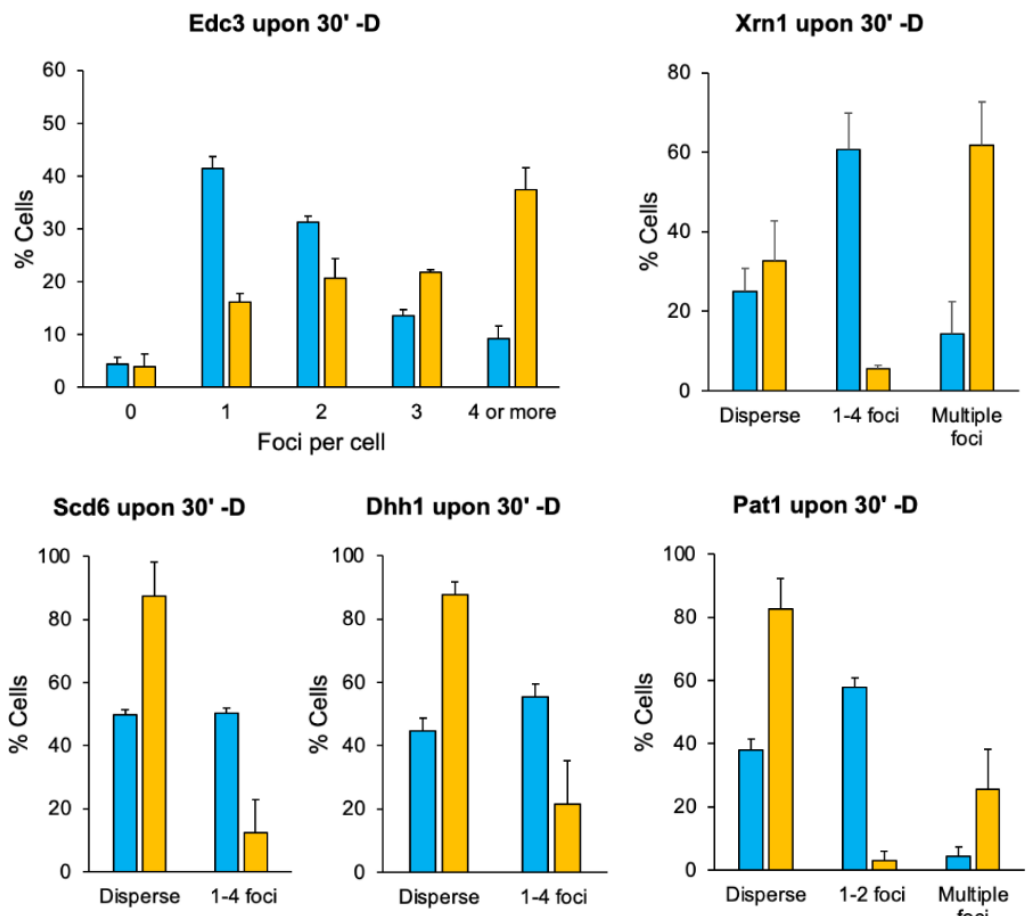

D

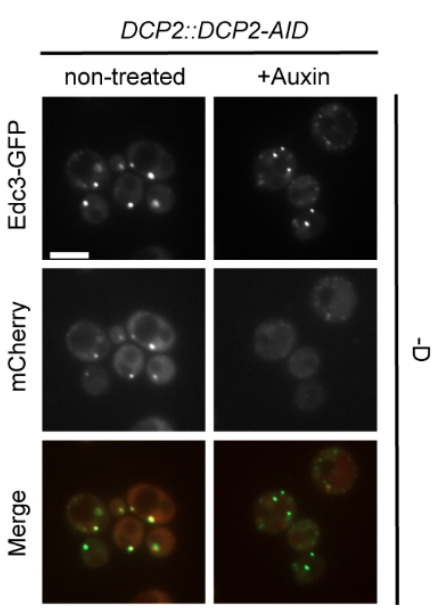

E
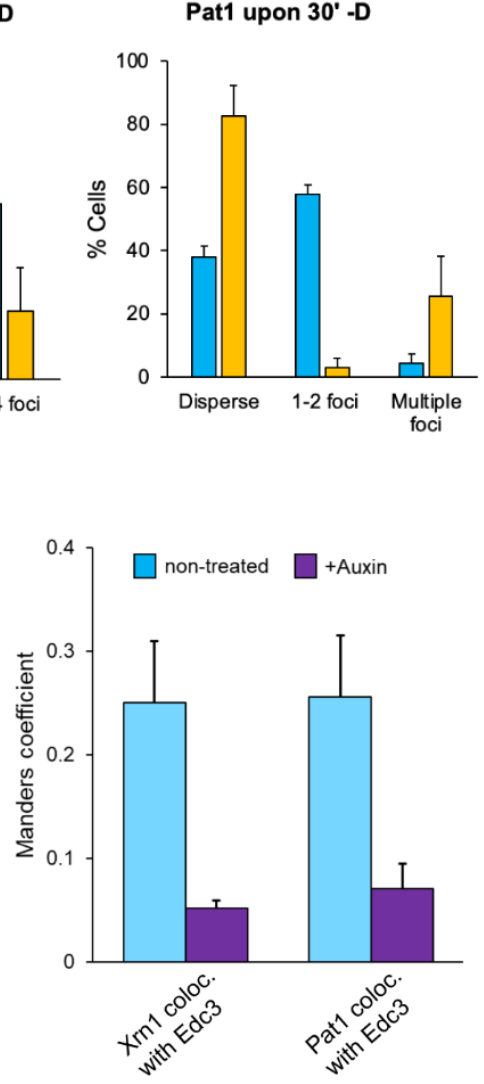
Figure 6. Dcp2 is essential for P-body assembly. A-B. Depletion of Dcp2 impairs $\mathrm{PB}$ formation. All strains were constructed from an isogenic background with $D C P 2$ genomically tagged with an auxin-inducible degron. The respective genes were either genomically appended with GFP or mCherry at the C-terminus (EDC3, SCD6 and $X R N 1$ ), or the respective mCherry-tagged protein was expressed from a low-copy plasmid on its endogenous promoter (Dhh1 and Pat1). Logarithmically growing cells were treated with $2 \mathrm{mM}$ auxin for $2 \mathrm{~h}$, then deprived from glucose for $30 \mathrm{~min}$ and imaged (A, scale bar $5 \mu \mathrm{m}$ ), and the number of mCherry foci was quantified (B). C-E. Dcp2 coordinates the recruitment of Xrn1 and Pat1 to Edc3 during PB formation. The strains expressing Edc3-GFP from panel 6A was genomically tagged with mCherry at the XRN1 locus (C) or transformed with a low-copy plasmid expressing Pat1-mCherry from its endogenous promoter (D). Logarithmically growing cells were treated as in panel 6A. Scale bar $5 \mu \mathrm{m}$. (E) Manders coefficients for the fraction Xrn1-mCherry or Pat1-mCherry colocalizing to Edc3-GFP upon 30 min -D. 


\section{Model}

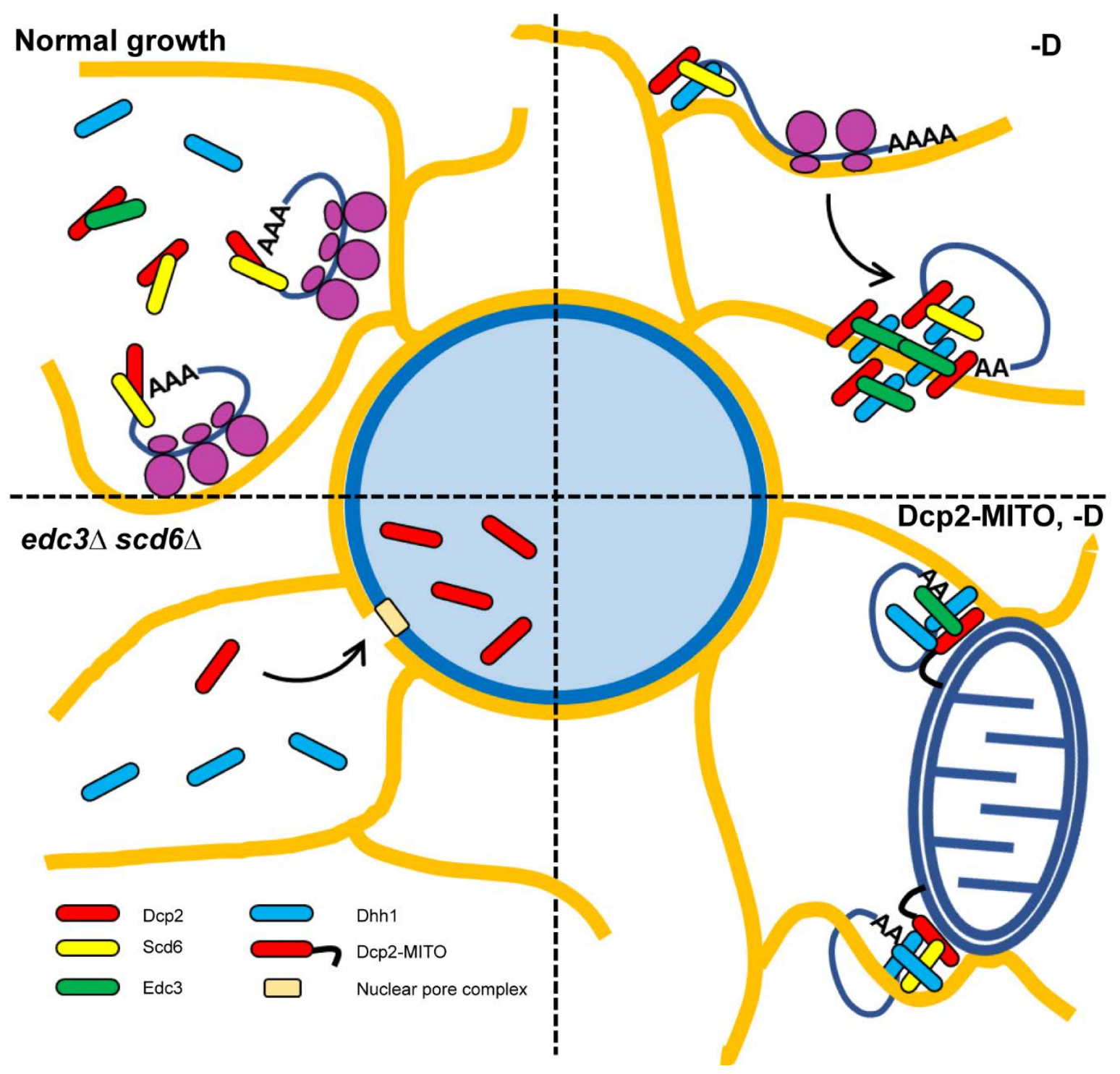


bioRxiv preprint doi: https://doi.org/10.1101/2020.09.07.285700; this version posted September 8, 2020. The copyright holder for this preprint (which was not certified by peer review) is the author/funder, who has granted bioRxiv a license to display the preprint in perpetuity. It is made available under aCC-BY 4.0 International license.

Figure 7. Schematic representation of our findings. For detailed information, see text. 